\title{
The Research and Implementation of Software Development Based on SOA Architecture
}

\author{
Yuhong Yang \\ Heilongjiang College of Construction
}

Keywords: SOA architecture, Web service, CMI code reuse; Function analysis; CMI

\begin{abstract}
This paper mainly is based on the loophole and the insufficiency of traditional different object oriented software development framework and code reuse, analyzing the advantage model structure of the service software system. Combining with referring to the actual accurate computer assisted instruction (CMI) application, it's speculated how to use Web services to realize further implementation of SOA architecture in the application of computer system. In addition, refinement technical index of the internal development of SOA architecture CMI system is further studied: system structure mode, server allocation and memory allocation and code reuse, SOA system for higher quality and stability.
\end{abstract}

\section{Introduction}

In recent years, with the deepening of software development approach research in our country, the applied and developed distributed extension technology enters the permeation era of the whole field. The business system establishment of different development platform and differentiated development tools makes the software systems of each enterprise increasingly complex, and the scale is also growing. The application function of the operating system structure has binding, which makes it hard for the compatibility and parallel of the dispersed independent system. Therefore, in order to better use of existing module, improve the matching efficiency, and speed up the development of software, reuse modules must be separated from the old system and incorporated into the new system, namely the SOA (Service Oriented Architecture) software design method. The distributed system that is constructed based on the method of routing services organization will have the advantages of architecture model and programming model at the same time, that can be used in software mergers and the subsequent development work.

\section{Software Development Based on SOA}

Because this paper mainly adopts the object-oriented software usage method provided by SOA to gradually build a loosely coupled distributed system, so that the overall level of reuse and expansibility can reach a high degree. Then, the paper draws up its focus direction and viewing angle difference for further research and analysis of SOA software development approach.

The Object-oriented Division of System. Based on SOA loose coupling software architecture characteristics, independent reusable method can be adopted to form system basis functions. For the external, the service function has clear interface that can achieve the call of transparent decoupling interface, completing revision adjustment operation.

The main components of service-oriented system are divided into three types: (1) Serving the client

It needs to use service applications or other forms of access to inquire from registry service center, then carry out the subsequent binding check work according to the regulations of transport protocol and service programs, and then realize the service function.

(2) Serving the provider

Essentially, it belongs to the main body of creating service and holds the service resources and distribution rights. Based on the service request from the client, service object's demand is executed remotely. It's usually adopted through the way to send the service interface information to the registry center, to get the consumption demand and potential users. 
(3) Serving the registry center

It's in the transfer position between the 2 items above. It has display service and provides the function of mutual information. It serves the clients through the query of registration information in service registry database, to get the interested or needed interface information. The publishers will effectively promote their service resources through the registry platform.

Service Oriented Architecture. Next, the sequence of operation for completing service oriented architecture: publishing, discovering, binding, and calling. Basically, these processes can ensure the service access information can be called by publishers and make timely information interaction, to position corresponding fit service standard through the registry center. Then in the subsequent information description, the service function is called further according to clients' data, to achieve the ultimate business requirement.

Because service belongs to the underlying architecture in SOA at more abstract level, so the service dynamic process of the demand should be defined. At the same time, SOA basic services must satisfy the demand of comprehensive and independent deployment, enabling system separating from other system and completing the whole process alone. Therefore, compared with the traditional way of development, SOA exerts its loose and coupled features to complete service sharing and information interaction under the standard.

In addition, SOA is easy to integrate existing system, and can insure to complete system and application service transition based on the original system settings. SOA also has a standardized framework, and can let each functional components work in parallel in the same good SOA system, and is convenient to add deployment follow-up service and infrastructure. In addition, SOA development way has quite high execution efficiency, and based on the reuse features, it can fully complete lifecycle assessment in the design, development, testing, and deployment of new software, significantly reducing the error rate and development cycle. At the same time, it can let orderly system keep good structure, which is easy for subsequent operation maintenance, to reduce the cost of second development.

\section{SOA Technology Implementation--Web}

Because the current SOA technical service sends and receives information through the network interface, the potential consumers can use information interface, with the aid of network service invocation to improve the utilization rate of SOA. Based on the flexible characteristic of Web service, it can provide natural advantages for SOA services, realizing highly open and flexible distribution of SOA.

Web Key Technology. SOA is bound by specific technical system, and it can work in multiple platforms (Web services, RMICOM, CORBA). Thus, broadly speaking, the technology replication can be directly realized on the Web. Strictly speaking, however, it also needs neutral agreement technology as dispensing. Web services and SOA features combining will have better reliability, ductility and openness, which will establish dominant position for SOA technology.

First of all, WSDL UDDI registry of SOA service information is implemented based on basic technology of Web services stacks (Fig. 1). Among them, the SOAP (Simple Object Access Protocol) is a kind of fixed message with XML format based on XML light communication protocol, transmitted through Web network. Generally, the message is packaged through SOAP envelope element, which is combined with an optional SOAP element head (header) and the element body. WSDL (Web Services Description Language) is also based on the XML message language, and it can transmit the analysis method and using rule provided by other application procedures completely through Web services. The UDDI defines the format of XML, and it can satisfy the identification and issue of different functions through different details adjustment. 


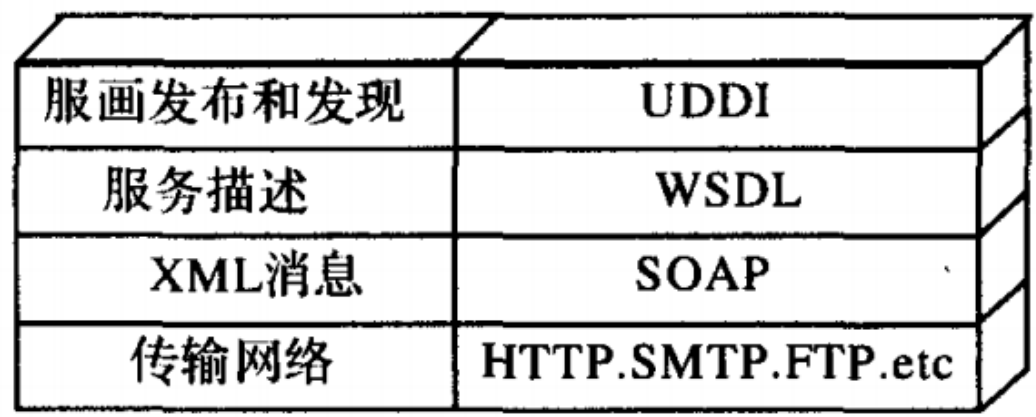

\begin{tabular}{|l|l|}
\hline Service issuance and discovery & UDDI \\
\hline Service description & WSDL \\
\hline SML message & SOAP \\
\hline Transmission network & HTTP.SMTP.FTP.etc \\
\hline
\end{tabular}

Figure 1. Web service protocol stack

Running of Service Data. After that, service demander can get service information through access of registry center, further to get service related WSDL document, which contains all the interactive information from Web to service, to achieve better data expression and transmission. At the same time, it also can complete that the demanders send SOAP request information to the provider sends and subsequent Web SOAP message return.

\section{Development of Application Instance}

Computer Management System Analysis. According to requirements of the available national remote education standard for the interactive operation of teaching management: (1) diversified curriculum is set up, namely the course can work in different CMI system. (2) Different platform development course can consolidate into a CMI system. (3) Course can easily move between different CMI systems. (4) Data is concise, regular, and easy to students for data analysis.

Based on the above requirements and the demand for open and interactive operation by SOA and the sharing background of service module and platform, the core concept of SOA service reuse rate needs to be built up gradually. It can make different systems realize data integration and resource sharing through the existing applications by reprograming the system based on it. At the same time, data transfer and data analysis can be convenient based on the loose coupling and distribution of SOA architecture.

In addition, it can be concluded through the analysis of characteristics CMO system that CMI system data mainly comes from students' basic information. Both in the examination system, and in the school educational administration management system, information storage classification function is indispensable. The general information needs to be stored in different function system, which increases the workload and transfer cost. Therefore, under the situation with the same need of multiple modules in different systems, SOA architecture can be complete function "copy" well. "Code reuse" is the development skill often used in SOA system. Based on the object-oriented software development, complicated problems of common data can be solved through code copy and instantiation analysis. So in CMI calculation education system, high frequency of "students' basic information", the encapsulated service data only needs to be called, complex interactive task of data can be completed, which is an effective solution.

CMI System Construction Based on SOA. Considering that Java development has very high compatibility and operability crossing different platforms, and at the same time, Web service function provided by J2EE capabilities can be very convenient to complete all the existing access requirements and information processing of service business, so using J2EE technology to carry out CMI system based on SOA system development. A preliminary framework is as follows: 


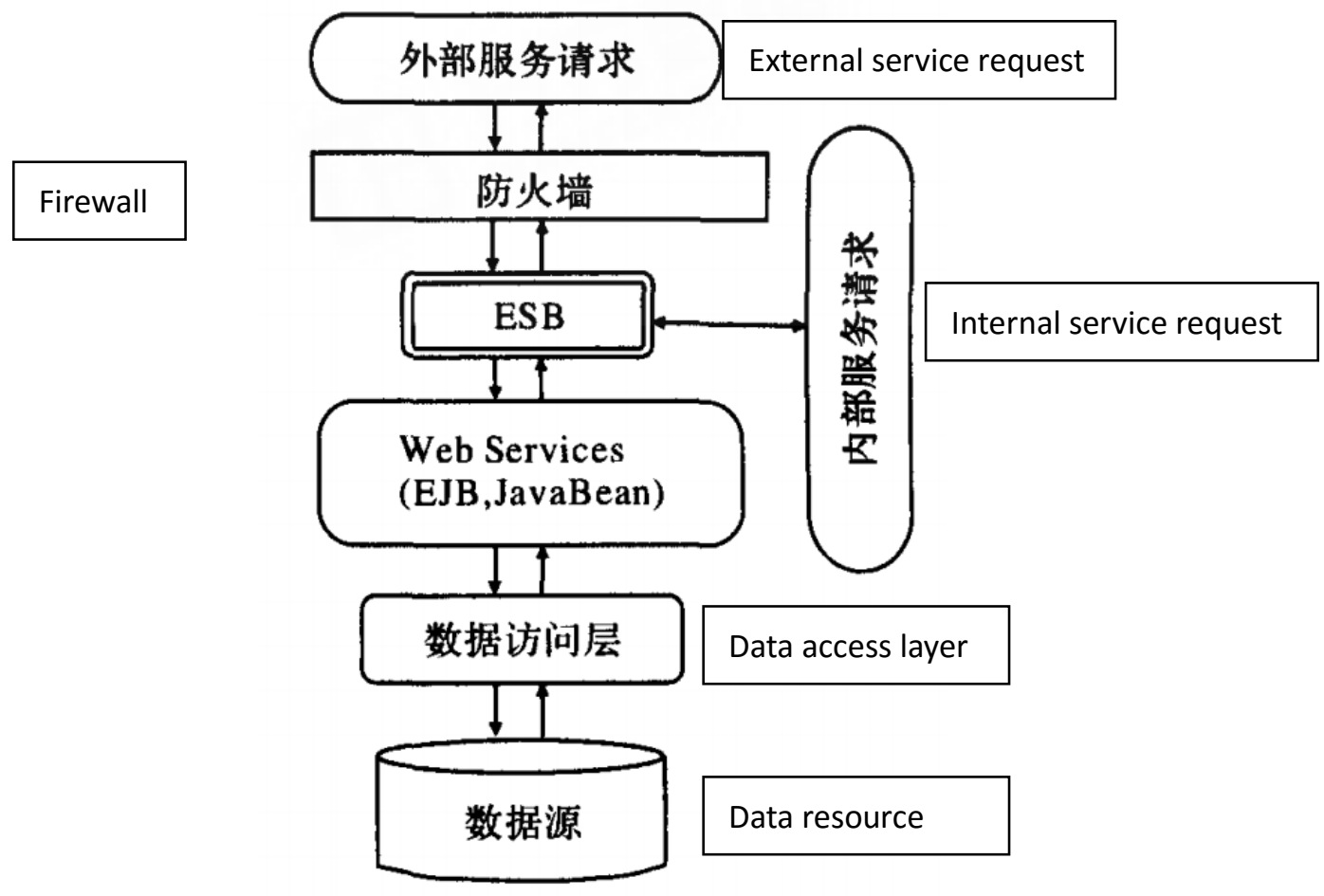

Figure 2. CMI system framework

The processing through the presentation layer includes: related programming data access of interface interaction components and browser and other procedures, implementing different service requests delivery. As the network security center, firewall wall establishes information counterguard between the risk area and local area network (LAN) to monitor network data communication, and strictly control access, only to allow security information pass and prevent illegal data invasion. ESB realizes the effect of data bus, using SOAP/HTTP/WSDL to realize the communication and service interaction function, and reserve security areas to strengthen the internal data security, synchronously improving service quality and efficiency of information processing. Web service layer implements information interaction of services and consumer through wireless session, effectively transferring Web services, and to send information back in time.

Implementation of CMI Function. With the situation that multiple systems are registered by students, Web creation service of specific into Java in CMI system is discussed as following, to achieve the function described data communication.

Firstly, WSAD built-in Web services wizard is started to pack the data objects as Web service, a number of tests can be performed during this period. Web service will be published to the UDDI, through client agent, to create and publish a Web service. After that, WSAD will automatically generate WSDL document, describing and recording the general method of Web service. The root elements definitions, message keep the key variable message of service delivery, and portType refers to the transmit mode of message. Binding element keeps interaction information. Service element stipulates the URL value to call Web Service value, which will be changed into the accessible address in publishing.

After the Web service code generated, Web service will need to be put in library with the client. Different from the name reference rule in EJB client, Web service client only needs to read Web service WSDL file registered by students, student information can be input through RPC. So, a loose coupling CMI teaching management system based on SOA architecture is completed.

\section{Conclusion}

SOA architecture represents a new software structure with standardization and liberalization. High loose coupling performance and reusability let it cannot be confined to a system. With the 
development of Web service technology, SOA multiple-platform heterogeneous leap can be realized. It is bound to become a mainstream of next generation of software architecture and development technology, and will be applied to more technical implementation and application support.

\section{References}

[1] Chen Xiaojiang Software Development Method Based on SOA Architecture, [J] Microelectronics and Computers, 2015 (22)

[2] Wei Dong Development And Implementation of SOA Architecture Software [D] Northwestern University, Department of Computer Science, 2009

[3] Liu Qiaojia. Intrusion detection technology in the application of the computer database analysis [J]. Journal of wireless technology, 2012, 10 (5): 117-119.

[4] Qin Liang. The computer database of shallow intrusion detection technology [J]. Computer knowledge and technology, 2013, 7 (3): 555-559.

[5] Guo paris-isvara. Virtual reality technology in the application of computer teaching experiment research [J]. Journal of jiangxi college of education, 2013 practices: 26-29. 